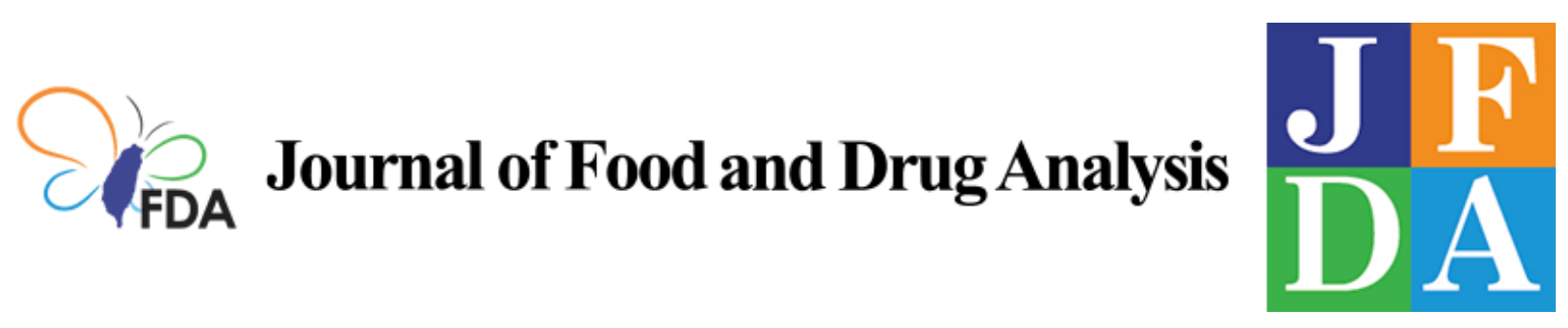

Volume 28 | Issue 4

Article 13

2020

\title{
Acknowledgment of reviewers
}

Follow this and additional works at: https://www.jfda-online.com/journal

Part of the Food Science Commons, Medicinal Chemistry and Pharmaceutics Commons,

Pharmacology Commons, and the Toxicology Commons

(c) (i) (8)

This work is licensed under a Creative Commons Attribution-Noncommercial-No Derivative Works 4.0 License.

\section{Recommended Citation}

Chiu, Lily (2020) "Acknowledgment of reviewers," Journal of Food and Drug Analysis: Vol. 28 : Iss. 4 , Article 13.

Available at: https://doi.org/10.38212/2224-6614.3232 


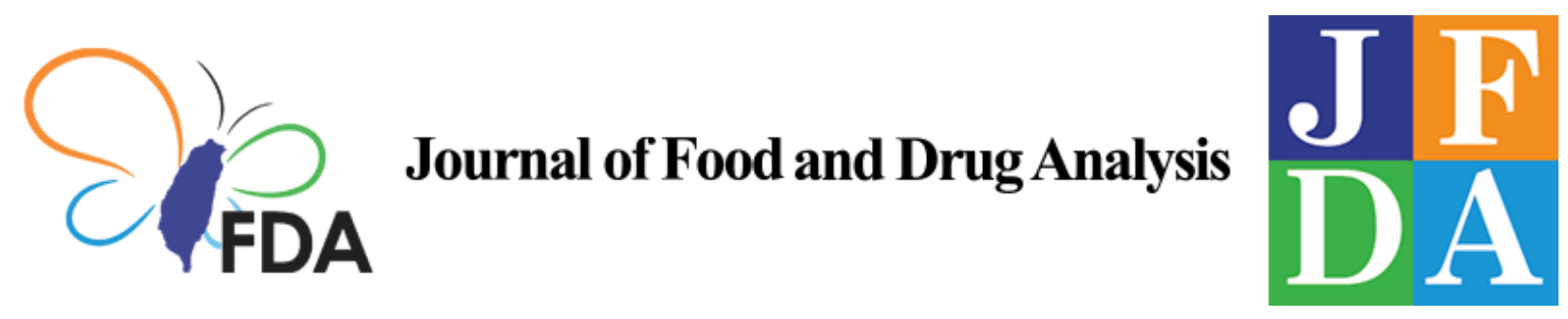

Volume 28 | Issue 4

Article 13

2020

Acknowledgment of reviewers

Follow this and additional works at: https://www.jfda-online.com/journal

Part of the Food Science Commons, Medicinal Chemistry and Pharmaceutics Commons,

Pharmacology Commons, and the Toxicology Commons

(c) (i) (8)

This work is licensed under a Creative Commons Attribution-Noncommercial-No Derivative Works 4.0 License.

\section{Recommended Citation}

Chiu, Lily (2020) "Acknowledgment of reviewers," Journal of Food and Drug Analysis: Vol. 28 : Iss. 4 , Article 13.

Available at: https://doi.org/10.38212/2224-6614.3232

This Acknowledgment of Reviewers is brought to you for free and open access by Journal of Food and Drug Analysis. It has been accepted for inclusion in Journal of Food and Drug Analysis by an authorized editor of Journal of Food and Drug Analysis. 


\title{
Acknowledgment of reviewers
}

\author{
Editors' note
}

The Editors and the Editorial Board of the Journal of Food and Drug Analysis would like to express their grateful appreciation to the scientific reviewers listed below for their careful and thoughtful efforts to enhance the quality of the journal.

Agrawal, Surendra S
Bai, Nai-Sheng
Bento, Ricardo Rodri-
gues de França
Bisgin, Abdullah Taner
Caddeo, Carla
Chan, Yin-Ching
Chang, Chia-Chuan
Chang, Chi-I
Chang, Chuang-Rung
Chang, Fang-Rong
Chang, Li-Chien
Chang, Lin-Chau
Chang, Wen-Te
Chang, Yuan-Shiun
Chang, Yu-Wei
Chang, Chia-Che
Chao, Hsi-Chun
Chao, Pei-Dawn Lee
Chau, Lai-Kwan
Chen, Bin-Huei
Chen, Chia-Yang
Chen, Chien-Fu
Chen, Chin-Shuh
Chen, Guan-Yuan
Chen, Hong-Jhang
Chen, Hsin-Chun
Chen, Hui-Ting
Chen, Ih-Sheng
Chen, Jemkun
Chen, Rong-Jane
Chen, Sung-Fang
Chen, Wen-Ling
Chen, Yen-Ling
Chen, Yi-Chen
Chen, Yu-Chih
Chen, Yu-Kuo
Chiang, Chang-Kang
Chiang, Cheng-Kang
Chiang, Chiao-Hsi
Chiang, Hsiu-Mei
Chang

Chiang, Meng-Tsan
Chiou, Yi-Shiou
Chiu, Hui-Wen
Chiu, Shih-Jiuan
Chou, Chen-Hsi
Chuang, Kuo-Hsiang
de Menezes, Irwin Rose
Alencar
Deng, Hua
Dinç, Erdal
El-Shazly, Mohamed
Esposito, Francesco
Fang, Jia-You
Faraji, Mohammad
Fararjeh, Abdulfattah
Salah
Fu, Wen-Mei
Gean, Po-Wu
Guh, Jih-Hwa
Gumustas, Mehmet
Guo, Lei
Haron, Mona
He, Xiaobo
Ho, Chi-Tang
Ho, Yuan-Soon
Hong, Wei
Hou, Wen-Chi
Hou, Yu-Chi
Hsieh, Chang-Wei
Hsieh, Pei-Wen
Hsu, Chin-Lin
Hsu, Pang-Hung
Hu, Cho-Chun
Hua, Kuofeng
Huang, Bu-Miin
Huang, Chih-Ching
Huang, Hui-Chi
Huang, Huisuo
Huang, Nai-Kuei
Huang, Rwei-Fen
Huang, Shih-Yi

Huang, Wei-Jan
Hung, Wei-Lun
Kao, Tsai-Hua
Kavitha, Sharma
Kumari
Ke, Liang-Yin
Kong, Kien-Voon
Kosir, Iztok Jože
Kuo, Jen-Min
Kuo, Ching-Chuan
Kuo, Hsing-Chun
Kuo, Yao-Haur
Kwon, Sung-Won
Lai, Ching-Shu
Lai, Guan-Yi
Lay, Horng-Liang
Lee, Ching-Kuo
Lee, Der-Yen
Lee, Jen-Ai
Lee, Seung Ju
Lee, Tzong-Huei
Lee, Wen-Jui
Li, Shiming
Li, Chi-Ying
Li, Jing
Li, Mengxing
Li, Min
Li, Shaoping
Li, Xiaojiaoyang
Liao, Hsiao-Wei
Liao, Wei-Ssu
Liaw, Chih-Chuang
Lin, Bi-Fong
Lin, Chun-Mao
Lin, Tzu-En
Lin, Yang-Wei
Lin, Yun-Lian
Lin, Zong-Hong
Ling, Min-Pei
Ling, Yong-Chien
Liu, Hung-Wen

Liu, Shinghwa
Lu, Chi-Yu
Lu, Long-Sheng
Lu, Mei-Chin
Lu, Mei-Kuang
Lu, Yue
Mahadik, Kakasaheb
Ramoo
Musso, Maurizio
Ermanno
Navaee, Kian
Neves de Lima, Ádley
Antonini
Ni, Jie
Niu, Yunwei
Pan, Chorng-Liang
Pan, Min-Hsiung
Parr, Maria Kristina
Pianezze, Silvia
Ragno, Gaetano
Sathirakul, Korbtham
Schmidt, Mark S.
Sharma, Kumari Kavita
Sheen, Lee-Yan
Sheu, Shyang-Chwen
Shiao, Young-Ji
Shrestha, Prabin
Shyur, Lie-Fen
Šimúth, Jozef
Song, Jingyuan
Su, Cheng-Kuan
Su, Nan-Wei
Subudhi, Bharat
Bhusan
Taoukis, Petros
Ting, Yuwen
Tsai, I-Lin
Tsai, Chia-Fen
Tsai, Jimmy Yung-Hsiang
Tsang, Wei-Lung


Tung, Yen-Chen

Ueng, Yune-Fang

Wang, Ching-Chiung

Wang, Chin-Kun

Wang, Chun-Chi

Wang, David Sheng-

Yang

Wang, Hui-Chun

Wang, Li-Hsuan

Wang, Meng-Ting
Wang, Ying-Jan

Weaver, Connie M

Weng, Meng-Shih

Wiczkowski, Wieslaw

Wong, Hin-Chung

Wong, Tit-Yee

Wu, Bin-Nan

$\mathrm{Wu}$, Chang-Jer

Wu, Hsin-Lung

$\mathrm{Wu}$, Kuo-Chen
$\mathrm{Wu}$, Sheng-Nan

$\mathrm{Wu}$, Tian-Shung

$\mathrm{Wu}, \mathrm{Tzu}-\mathrm{Hua}$

$\mathrm{Wu}$, Wen-Mein

$\mathrm{Wu}$, Yang-Chang

$\mathrm{Wu}$, Yongning

$\mathrm{Wu}, \mathrm{Yu}$-Tse

Wybraniec, Slawomir

Xia, Qingsu

Xia, Wei
Xie, Wen

Xue, Jian

Yang, Meihua

Yang, Yu-Liang

Yen, Chia-Hung

Yoon, In-Soo

Yuan, Yuwei

Yuan, Zhiqin

Zhang, Liang

Zhang, Yongmei 